\title{
Sum-rule for large-Nc QCD and application to heavy quarkonia
}

\section{Citation}

Lee, Dean, and Howard Georgi. 1998. “Sum-Rule for Large-Nc QCD and Application to Heavy

Quarkonia." Physics Letters B 426 (3-4): 367-74. https://doi.org/10.1016/s0370-2693(98)00287-1.

\section{Permanent link}

http://nrs.harvard.edu/urn-3:HUL.InstRepos:41467472

\section{Terms of Use}

This article was downloaded from Harvard University's DASH repository, and is made available under the terms and conditions applicable to Other Posted Material, as set forth at http:// nrs.harvard.edu/urn-3:HUL.InstRepos:dash.current.terms-of-use\#LAA

\section{Share Your Story}

The Harvard community has made this article openly available.

Please share how this access benefits you. Submit a story.

\section{Accessibility}


\#HUTP-97/A057

$9 / 97$

\title{
Sum-rule for large- $N_{c}$ QCD and application to heavy quarkoniał
}

\author{
Dean Leef and Howard Georgi \\ Harvard University \\ Cambridge, MA 02138
}

\begin{abstract}
We introduce a new sum-rule for large- $N_{c}$ QCD which relates the density of heavy quarkonium states, the state-averaged square of the wavefunction at the origin, and the heavy quark current-current correlator. Focusing on the region of energy just above perturbative threshold, we calculate the correlator by incorporating arbitrarily high orders in the QCD coupling $\alpha_{s}$. We use the sum-rule to determine the bottomonium potential using experimentally measured s-wave leptonic widths and compare the result with the potential obtained by direct calculation from the measured $s$-wave spectrum. We discuss the utility of the sum-rule method for accurate determination of the confining potential.
\end{abstract}

\section{Introduction}

In the heavy quarkonium system, we can study quark confinement without the added complexity of relativistic processes. As the mass of the quark becomes large, the dynamics of the quarkonium system becomes non-relativistic and can be described by the Schrödinger equation with a static potential. The short distance behavior of this potential can be approximated by single gluon exchange and therefore has the familiar Coulombic form. In this paper, we consider the remaining long distance part of the potential, which we refer to as the confining potential. In the course of our analysis we answer the following questions: (i) Can the confining potential in some region of $r$ be determined from the spectrum by direct calculation, i.e., without parameter fits? (ii) Can the confining potential also be determined from other data, such as the s-wave leptonic widths $\Gamma_{e^{+} e^{-}}$? (iii) How do the results of (i) and (ii) compare, and can we accommodate both data sets with one potential?

\footnotetext{
${ }^{1}$ Research supported in part by the National Science Foundation under Grant \#PHY-9218167.

${ }^{2}$ Supported in part by the Fannie and John Hertz Foundation.
} 
To answer these questions we make use of the large- $N_{c}$ expansion, where $N_{c}$ is the number colors. We derive a sum-rule that relates the imaginary part of the heavy quark currentcurrent correlator with the product of $\rho$, the density of s-wave states, and $\left|\psi^{\text {ave }}(0)\right|^{2}$, the state-averaged square of the wavefunction at the origin. The derivation involves calculating the imaginary part of an integral of the heavy quark current-current correlator in momentum space. We do this calculation in two different ways, once using perturbative methods and then again using spectral sums. The sum-rule follows from equating the two calculations.

Sum-rules for the current-current correlator for light quark systems have a long history [囬]. Our approach is new in that we restrict our attention to heavy quark currents and focus on the energy region just above the perturbative $q \bar{q}$ threshold. As it turns out this energy region has some interesting characteristics. One feature is the appearance of threshold singularities in the perturbative calculation. To account for these singularities, our calculation will include contributions from arbitrarily large orders in $\alpha_{s}$, unlike more conventional treatments. Another feature is that the effects of confinement can be described using non-relativistic bound state methods. As we attempt to illustrate, application of the sum-rule provides a useful complement to non-relativistic methods. In particular, we discuss its use in the determination of the confining potential for the bottomonium system.

The organization of the paper is as follows. In the sections 2 and 3, we derive the sumrule. In section 2 , we discuss the perturbative calculation of the current-current correlator, and we note that it is straightforward to include information from arbitrarily high orders in $\alpha_{s}$ by using the energy spectrum of the Coulomb bound states. In section 3 , we calculate the current-current correlator as a spectral sum and relate it to the properties of the physical $q \bar{q}$ states in the large- $N_{c}$ limit. In section 4 , we test the sum-rule for the bottomonium system. In the remaining sections we discuss various ways to determine the confining potential, discuss our various approximations and the physics we have left out, and answer the questions (i), (ii), and (iii).

\section{Perturbative calculation}

We consider quantum chromodynamics with $N_{c}$ colors and one flavor of heavy quark, which we call $q$. Let $m$ be the mass of the heavy quark and $J^{\mu}(x)$ be the corresponding electric current. We can write the renormalized current-current correlator in momentum-space as

$$
\int d^{4} x e^{i k x}\left\langle 0\left|T\left[J^{\mu}(x) J^{\nu}(0)\right]\right| 0\right\rangle=\lim _{\varepsilon \rightarrow 0^{+}}\left(g^{\mu \nu}-\frac{k^{\mu} k^{\nu}}{k^{2}+i \varepsilon}\right) \Pi\left(\sqrt{k^{2}}, \varepsilon\right),
$$

where $\varepsilon$ is the infinitesimal positive parameter used to regulate the singularities of the bare propagators. Because we are in the time-like momentum region $k^{2}>0, k^{0}>0$, we can move to the rest frame where $\vec{k}=0$ and rewrite $\Pi\left(\sqrt{k^{2}}, \varepsilon\right)$ as $\Pi\left(k^{0}, \varepsilon\right)$. 
Let $z$ and $\Delta z$ be fixed positive numbers such that $\Delta z<<z<<1$. Let us define the integral

$$
P(m, z, \Delta z)=\lim _{\varepsilon \rightarrow 0^{+}} \int_{\zeta \in C(z, \Delta z)} \Pi((2+\zeta) m, \varepsilon) d \zeta,
$$

where $C$ is the contour in the complex $\zeta$-plane shown in Figure 1. The purpose of this contour integral will be explained in a moment when we discuss the $N_{c} \rightarrow \infty$ limit. Our choice of energy values places us within the non-relativistic $\bar{q} q$ resonance spectrum, just above the perturbative threshold, $k^{0}=2 \mathrm{~m}$.

If the dimensionless quantities $z$ and $\Delta z$ are held fixed, we expect $P$ to have a welldefined asymptotic expansion in $\alpha_{s}\left(m^{2}\right)$ as $m \rightarrow \infty$, where $\alpha_{s}\left(m^{2}\right)$ is the QCD coupling at scale $m^{2}$. In the following we abbreviate $\alpha_{s}\left(m^{2}\right)$ as $\alpha_{s}$. We are interested in the imaginary (absorptive) part of $P$, which we write as

$$
\operatorname{Im} P(m, z, \Delta z)=N_{c} \frac{m^{2}}{2 \pi} \Delta z \cdot G\left(z, \alpha_{s}\right)
$$

The perturbative expansion of $G$ will be discussed below. Let us now divide out the explicit $N_{c}$ in (3) and consider

$$
N_{c}^{-1} \operatorname{Im} P(m, z, \Delta z)
$$

in the simultaneous limit $N_{c} \rightarrow \infty, m \rightarrow \infty$. We rescale the coupling constant in the standard way so that $\alpha_{s} C_{F}$ has a well-defined limit as $N_{c} \rightarrow \infty$. We then have

$$
\lim _{N_{c} \rightarrow \infty}\left[N_{c}^{-1} \operatorname{Im} P(m, z, \Delta z)\right]=\frac{m^{2}}{2 \pi} \Delta z \cdot G\left(z, \alpha_{s}\right) .
$$

As $N_{c} \rightarrow \infty$, the $\bar{q} q$ resonances become stable and the corresponding pole singularities approach the real $\zeta$-axis from below. As $m \rightarrow \infty$, we expect the density of such pole singularities approaching the real $\zeta$-axis to increase and anticipate that the integrand in (2) will exist in the double limit $N_{c} \rightarrow \infty, m \rightarrow \infty$ only in the sense of a distribution. The contour integral defining $P$, however, does not have such problems. The pole singularities approach the real axis from one side and therefore do not pinch the contour. This is the motivation for our definition of $P$.

For $\alpha_{s} \ll z^{1 / 2}$, we can compute $G$ in (5) by expanding in powers of $\alpha_{s}$, i.e., the usual perturbative loop expansion. This expansion, however, is inadequate for $z^{1 / 2} \lesssim \alpha_{s}$. As the

\footnotetext{
${ }^{1}$ To avoid the possibility of endpoint singularities we have pulled the endpoints of the contour slightly off the real axis and into the upper half plane, i.e, we have added a small positive imaginary part to $z$. This technical point is of little consequence, however, since the limit $\operatorname{Im} z \rightarrow 0^{+}$presents no problems.

${ }^{2}$ As we will discuss section 6 , the precise scale at which $\alpha_{s}$ is evaluated does not matter to the order we are working. The difference between $\alpha_{s}\left(m^{2}\right)$ and $\alpha_{s}\left(z m^{2}\right)$ is not important.

${ }^{3}$ This is discussed in the next section.
} 
order of $\alpha_{s}$ increases, the order of the $z^{-1 / 2}$ singularity also increases [3]. It is therefore more useful to expand $G$ as

$$
G\left(z, \alpha_{s}\right)=\sum_{n=1}^{\infty}\left(\alpha_{s} C_{F}\right)^{n} \cdot G_{n}\left(\alpha_{s} C_{F} z^{-1 / 2}\right)
$$

where $C_{F}=\frac{N_{c}^{2}-1}{2 N_{c}}$. The leading term, $G_{1}$, is given by the sum of all simple ladder diagrams, which in turn can be obtained from the bound-state spectrum for the Coulomb potential. $G_{1}$ has been calculated explicitly in [4] (see also [2]), with the result

$$
G_{1}(x)=\frac{1}{x}+\frac{\pi}{2}+\frac{x}{2} \sum_{n=1}^{\infty} \frac{1}{n^{2}+\frac{x^{2}}{4}} .
$$

We will use this form in section 4 .

\section{Spectral sum calculation}

Let us now repeat the calculation in (5), this time using the spectral representation of the current-current correlator. We consider the contributions of all single and multiparticle states to the correlator:

$$
\begin{aligned}
\left\langle 0\left|T\left[J^{\mu}(x) J^{\nu}(0)\right]\right| 0\right\rangle & =\theta\left(x^{0}\right) \sum_{n}\left\langle 0\left|J^{\mu}(x)\right| n\right\rangle\left\langle n\left|J^{\nu}(0)\right| 0\right\rangle \\
& +\theta\left(-x^{0}\right) \sum_{n}\left\langle 0\left|J^{v}(0)\right| n\right\rangle\left\langle n\left|J^{\mu}(x)\right| 0\right\rangle .
\end{aligned}
$$

In the $N_{c} \rightarrow \infty$ limit, the coupling of the current to multiparticle meson states is suppressed by explicit factors of $N_{c}^{-\frac{1}{2}}$. The contribution of these states to the spectral sum in (8) is therefore suppressed by $N_{c}^{-1}$, and we ignore such states in the $N_{c} \rightarrow \infty$ limit.

Having reduced the correlator to a spectral sum over $\bar{q} q$ resonance states, we use this spectral sum to compute the imaginary part of $N_{c}^{-1} P$. In the $N_{c} \rightarrow \infty$ limit it is not necessary to include all resonance states. Resonances which do not approach the contour $C$ give an imaginary contribution that is suppressed by $N_{c}^{-1}$. f Let us use the index $r$ to label resonances which approach the contour as $N_{c} \rightarrow \infty$. For each resonance $r$, let $M_{r}$ be the mass, $\Gamma_{r}$ be the width, and $f_{r} g_{\mu \nu}$ be the coupling with the current. We then have

$$
N_{c}^{-1} \operatorname{Im} P(m, z, \Delta z)=\sum_{r} \operatorname{Im}\left[\int_{\zeta \in C(z, \Delta z)} \frac{-N_{c}^{-1}\left|f_{r}\right|^{2} d \zeta}{((2+\zeta) m)^{2}-\left(M_{r}-\frac{i}{2} \Gamma_{r}\right)^{2}}\right]+O\left(N_{c}^{-1}\right) .
$$

¿From here on, we suppress writing the $O\left(N_{c}^{-1}\right)$ error term. We can compute the integral in terms of the residues of the poles which approach $C$,

\footnotetext{
${ }^{4}$ The real contribution, however, is not suppressed.
} 


$$
N_{c}^{-1} \operatorname{Im} P(m, z, \Delta z)=\sum_{r} \frac{\pi N_{c}^{-1}\left|f_{r}\right|^{2}}{2(2+z) m^{2}} .
$$

Since these resonances are non-relativistic bound states, we can use the van-Royen-Weisskopf result,

$$
N_{c}^{-1}\left|f_{r}\right|^{2}=4\left|\psi_{r}(0)\right|^{2} M_{r}
$$

where $\psi_{r}(0)$ is the Schrödinger wavefunction of the resonance at the origin. Only s-wave states couple to the photon in this limit. Substituting (11) into (10), we get

$$
N_{c}^{-1} \operatorname{Im} P(m, z, \Delta z)=\frac{2 \pi}{m} \sum_{r}\left|\psi_{r}(0)\right|^{2} .
$$

Comparing (5) and (12), we find

$$
\frac{2 \pi}{m} \sum_{r}\left|\psi_{r}(0)\right|^{2}=\frac{m^{2}}{2 \pi} \Delta z \cdot G\left(z, \alpha_{s}\right)
$$

The spectral sum on the left side of (13) is a discrete sum. It therefore has intrinsic fluctuations of size $[\Delta n(z m)]^{-1}$, where $\Delta n(z m)$ is the number of s-wave states between $(2+z) m$ and $(2+z+\Delta z) m$. These fluctuations are not reproduced in the perturbative calculation at any finite order in $\alpha_{s}$. This is what we expect from an operator product analysis in which the fluctuations are associated with matrix elements of higher dimension operators, proportional to powers of $\Lambda_{\mathrm{QCD}}$ [5]. We conclude that $[\Delta n(z m)]^{-1}$ is smaller than any power of $\alpha_{s}$ as $m \rightarrow \infty$.

Let $\rho(z m)$ be the density of s-wave states at energy $z m$. We can rewrite

$$
\frac{1}{m} \sum_{r}\left|\psi_{r}(0)\right|^{2} \rightarrow \rho(z m)\left|\psi_{z m}^{\mathrm{ave}}(0)\right|^{2} \Delta z
$$

where $\left|\psi_{z m}^{\text {ave }}(0)\right|^{2}$ is the average of $\left|\psi_{r}(0)\right|^{2}$ for resonances with energies near $z m$. We then have

$$
2 \pi \rho(z m)\left|\psi_{z m}^{\mathrm{ave}}(0)\right|^{2}=\frac{m^{2}}{2 \pi} G\left(z, \alpha_{s}\right)
$$

or

$$
\rho(z m)=\frac{m^{2} G\left(z, \alpha_{s}\right)}{4 \pi^{2}\left|\psi_{z m}^{\operatorname{ave}}(0)\right|^{2}} .
$$

At this point, the careful reader may wonder whether we have done something inconsistent, because we have argued in this section that the physical $q \bar{q}$ bound states affect the imaginary part of the current-current correlator only if they approach the contour $C$, while in section 2, we saw that the perturbative calculation of the same imaginary part receives contributions which appear to be associated with the Coulomb bound states below perturbative threshold, not on the contour. The difference stems from the presence of the continuum 
states in the perturbative calculation. The $\sqrt{z}$ singularity from the continuum states can be distorted by the far-away poles. Effectively, the contribution to the imaginary part of the correlator in (5-7) is a product of the real part of the Coulomb bound state poles with the imaginary part from the continuum states.

\section{Bottomonium test}

In this section we evaluate the right and left sides of the sum-rule (16) for bottomonium states. We begin with the right side. For our purposes we use the approximation

$$
G\left(z, \alpha_{s}\right) \approx \alpha_{s} C_{F} \cdot G_{1}\left(\alpha_{s} C_{F} z^{-1 / 2}\right)
$$

where $G_{1}$ is defined in (可), with values $m_{b}=4600 \mathrm{MeV}$ and $\alpha_{s}\left(m_{b}^{2}\right) C_{F}=0.28$. The stateaveraged square of the wavefunction at the origin, $\left|\psi_{z m}^{\text {ave }}(0)\right|^{2}$, can be determined from the leptonic widths of the s-wave states. The relation is

$$
\left|\psi_{z m}^{\mathrm{ave}}(0)\right|^{2}=\frac{\Gamma_{e+e-} M^{2}}{16 \pi Q^{2} \alpha_{e m}^{2}},
$$

where $\Gamma_{e+e-}$ is the decay width into $e^{+} e^{-}, M$ is the mass of the meson (i.e., $2 m+z m$ ), and $\alpha_{e m}$ is the electromagnetic coupling.

Let us define $E=z m$. The left side of (16) can be obtained in the following manner. We first define a discrete function $n(E)$ such that $n\left(E_{1 s}\right)=1, n\left(E_{2 s}\right)=2, \cdots$, where $E_{k s}$ is the energy of the $k^{t h}$ s-wave meson. We smoothly interpolate between the values $E_{k s}$, thereby extending $n(E)$ to a differentiable function. We then differentiate $n(E)$ to get the density of states, which we call $\rho_{1}(E)$. We will be working with several versions of the densities of states and it is necessary to give them different names. Let us define $\rho_{2}(E)$ as the right side of $(16)$,

$$
\rho_{2}(E)=\frac{m^{2} G\left(z, \alpha_{s}\right)}{4 \pi^{2}\left|\psi_{E}^{\operatorname{ave}}(0)\right|^{2}} .
$$

In Figure 2 we have plotted $\rho_{1}(E)$ and $\rho_{2}(E)$ as functions of the meson mass $2 m+E$. The results indicate that the sum-rule is satisfied rather well.

\section{Determination of the potential}

In this section we propose several new methods for calculating the bottomonium confining potential. The sum-rule (16) gave us two calculations for the density of s-wave states, $\rho_{1}$ and $\rho_{2}$. In this section we use WKB methods to convert $\rho_{1}$ and $\rho_{2}$ into their corresponding potentials. 
Let us write the potential for the heavy quarkonium system as

$$
V(r)=-\frac{\alpha_{s} C_{F}}{r}+U(r)
$$

We now take a moment to discuss the expected behavior of $U(r)$. In section 4 we found that the number of s-wave states with energy less than $z m$ grows more rapidly than any inverse power of $\alpha_{s}$ as $m \rightarrow \infty$. The number of nodes of the s-wave wavefunctions with energy $z m$ must then scale faster than any inverse power of $\alpha_{s}$. This implies that $U(r)$ is slowly varying on distance scales of size $m^{-1} z^{-\frac{1}{2}}$, the approximate distance between nodes. We also expect that the magnitude of $U(r)$ is small near $r=0$. This is because the leading term in the perturbative calculation, $\alpha_{s} C_{F} \cdot G_{1}\left(\alpha_{s} C_{F} z^{-1 / 2}\right)$, is produced by the continuous spectrum of the Coulomb potential. The bound states of $V(r)$ must therefore approximate the continuous states of the Coulomb potential and the contribution from $U(r)$ near $r=0$ must be small.

For the purposes of calculating energy levels and wavefunctions, we can ignore the change of $U(r)$ on length scales $\Delta r \lesssim m^{-1}$ and approximate $V(r)$ by $V_{I}(r)$ :

$$
V_{I}(r)=-\frac{\alpha_{s} C_{F}}{r}+U\left(r+m^{-1}\right)
$$

This approximation introduces an error of size $\frac{1}{E \cdot M} \frac{d U}{d r}$.

Since $U\left(m^{-1}\right) \ll E \ll m$, we can subtract a constant term, $U\left(m^{-1}\right)$, from $V$ by a small redefinition of the heavy quark mass

$$
\begin{gathered}
m \rightarrow m+\frac{1}{2} U\left(m^{-1}\right) \\
V_{I} \rightarrow V_{I I}=V_{I}-U\left(m^{-1}\right) .
\end{gathered}
$$

This shift introduces an error on the wavefunctions and masses of the meson states of size $\frac{1}{m} U\left(m^{-1}\right)$. Combining (23) with (21), we have

$$
V_{I I}(r)=-\frac{\alpha_{s} C_{F}}{r}+W(r)
$$

where

$$
W(r)=U\left(r+m^{-1}\right)-U\left(m^{-1}\right)
$$

We now apply the WKB approximation. In the WKB approximation the density of states is given by

$$
\rho(E)=\frac{\sqrt{m}}{\pi} \int_{0}^{V_{I I}^{-1}(E)} \frac{d y}{2 \sqrt{E-V_{I I}(y)}}
$$


where the size of the error is $n(E)^{-1}$. The Coulomb term effects the integrand of $(\sqrt[26]{)})$ only in the small region $0 \leq y \lesssim E^{-1}$. As a result, the Coulomb term makes only a small contribution to $\rho(E)$ of size $\alpha_{s}$. We ignore this contribution and approximate $\rho(E)$ as

$$
\rho(E) \approx \frac{\sqrt{m}}{2 \pi} \int_{0}^{W^{-1}(E)} \frac{d r}{\sqrt{E-W(r)}} .
$$

Let us define a function $F(x)$ implicitly as

$$
\frac{1}{F(W(r))}=\frac{d W(r)}{d r},
$$

where we have assumed that $W(r)$ is an increasing function of $r$. We then have

$$
\rho(E) \approx \frac{\sqrt{m}}{2 \pi} \int_{0}^{E} \frac{F(Y) d Y}{\sqrt{E-Y}}
$$

which we recognize as an Abel transform of $F$. We can invert the transform,

$$
F(E)=\frac{2}{\sqrt{m}} \frac{d}{d E} \int_{0}^{E} \frac{\rho(Y) d Y}{\sqrt{E-Y}} .
$$

Combining (28) and (30) and integrating, we find

$$
r(W)=\frac{2}{\sqrt{m}} \int_{0}^{W} \frac{\rho(Y) d Y}{\sqrt{W-Y}} .
$$

This gives us the inverse of $W(r)$. Using $\rho_{1}$ and $\rho_{2}$ from the previous section, we can calculate the corresponding potentials $W_{1}$ and $W_{2}$. Doing this calculation, we find that $W_{1}$ and $W_{2}$ agree quite well. The results are shown in Figure 3. Purely for comparison, we plot $\alpha_{s} C_{F} / r$, the negative of the Coulomb potential, to emphasize that it is not important compared to the confining potential in most of the region of interest.

\section{Discussion}

The large- $N_{c}$ sum-rule provides a new perspective on the heavy quarkonium confining potential by drawing an explicit relation between the density of states and the wavefunction at the origin. As we have seen, the confining potential can now be determined in two different ways. In this section we discuss our approximations and consider the question of how to use this new information to determine the confining potential more accurately.

We have made two approximations in the derivation of our sum-rule, large $N_{c}$ and small $\alpha_{s}$. We do not believe that the large $N_{c}$ approximation introduces a very serious error in this analysis. It is used to argue that the bound-state poles are close to the real axis. Because 
the states we actually use in our analysis are rather narrow, this is probably reasonable. By working to first order in $\alpha_{s} C_{F}$ in (6), we are ignoring a number of potentially important effects, for example the running of the coupling. Thus, for example, we cannot say whether we should evaluate the coupling at $m^{2}$, as we have done, or at a smaller mass scale like $E^{2}=(z m)^{2}$. We are thus ignoring terms proportional to powers of $\alpha_{s} \ln (1 / z)$. We must do this for our perturbation series to be consistent. Because we work to all orders in $\alpha_{s} / \sqrt{z}$, we can go down to $z$ for which this is of order one, but for $\alpha_{s} \ln (1 / z)$ to be large requires still smaller $z \approx e^{-B / \alpha_{s}} \approx \Lambda_{Q C D} / m$, which implies $E=z m \approx \Lambda_{Q C D}$. We expect perturbation theory to break down in this region.

Our calculation of $W_{1}$ and $W_{2}$ was done by means of integral transform methods, rather than parameter fitting. We believe this carries certain advantages, such as calculational simplicity and the elimination of initial biases for the behavior of the potential. Our results for $W_{1}$ and $W_{2}$ appear to indicate a logarithmic or small fractional power law behavior for the confining potential in the relevant region for the observed bottomonium spectrum $\left(r \lesssim 10^{-2}\right.$ $\mathrm{MeV}^{-1}$ ). There are several ways to use our results to produce a more accurate representation of the confining potential. One approach is to use $W_{1}$ or $W_{2}$ or some average of the two as an initial result and then use numerical fitting to find the best possible correction. We expect this correction to be quite small, however, since as we now show the initial result appears to be quite accurate already.

We incorporate data for both the s-wave energy levels and leptonic widths in the most simple way by constructing an average density of states,

$$
\rho_{\text {ave }}(E)=\frac{\rho_{1}(E)+\rho_{2}(E)}{2} .
$$

We then use (31) to find the corresponding potential for $\rho_{\text {ave }}(E)$. As before we have taken

$m_{b}=4600 \mathrm{MeV}$ and $\alpha_{s}\left(m_{b}^{2}\right) C_{F}=0.28$. We now put the Coulomb term back in and calculate the resulting spectrum and leptonic widths. The results are shown in Table 1.

\section{$\begin{array}{ll}7 & \text { Summary }\end{array}$}

In our analysis of the heavy quarkonium confining potential we have answered the three questions posed in the introduction, and the answer to each question is positive. We derived a sum-rule that relates the imaginary part of the heavy quark current-current correlator with the product of the density of s-wave states and the state-averaged square of the wavefunction at the origin. As we have seen, application of the sum-rule provides a useful complement to non-relativistic potential methods. 


\section{Acknowledgments}

We are grateful to S. Coleman, A. Hoang, R. Marshall, A. Radyushkin and M. Voloshin for helpful discussions and suggestions.

\section{References}

[1] E.C. Poggio, H.R. Quinn, S. Weinberg, Phys. Rev. D13 (1976) 1958; V.A. Novikov, L.B. Okun, M.A. Shifman, A.I. Vainshtein, M.B. Voloshin, V.I.Zakharov Phys. Rept. 41 (1978) 1. See particularly [2] where a related approach is taken, but with somewhat different conclusions.

[2] M.B. Voloshin, Nucl.Phys. B154 (1979) 356.

[3] This observation led Appelquist and Politzer to the prediction of charmonium - T. Appelquist, H.D. Politzer, Phys. Rev. Lett. 34 (1975) 43; Phys. Rev. D12 (1975) 1404.

[4] V. Fadin, V. Khoze, Sov. J. Nucl. Phys. 48 (2), (1988) 309.

[5] M. A. Shifman, A. I. Vainshtein and V. I. Zakharov, Nucl. Phys. B147 (1979) 385, 448. 
Table 1

$\begin{array}{lllll}\text { Level } & \text { Mass (th.) } & \text { Mass (ex.) } & \Gamma_{e^{+} e^{-}} \text {(th.) } & \Gamma_{e^{+} e^{-}} \text {(ex.) } \\ \text { 1s } & 9490 \mathrm{MeV} & 9460 \mathrm{MeV} & 1.3 \mathrm{keV} & 1.3 \mathrm{keV} \\ 2 \mathrm{~s} & 10040 & 10020 & .63 & .52 \\ 3 \mathrm{~s} & 10370 & 10360 & .44 & .47 \\ 4 \mathrm{~s} & 10600 & 10580 & .34 & .59 \\ 5 \mathrm{~s} & 10820 & 10860 & .34 & .31 \\ 6 \mathrm{~s} & 11000 & 11020 & .17 & .13 \\ 2 \mathrm{p} & 9900 & 9890 & & \\ 3 \mathrm{p} & 10260 & 10260 & \end{array}$

\section{Figure Captions}

Figure 1. Plot of the integration contour $C$.

Figure 2. Plot of the densities $\rho_{1}$ and $\rho_{2}$.

Figure 3. Plot of the confining potentials $W_{1}$ and $W_{2}$. The dotted line is $\alpha_{s} C_{F} / r$. 
Figure 1

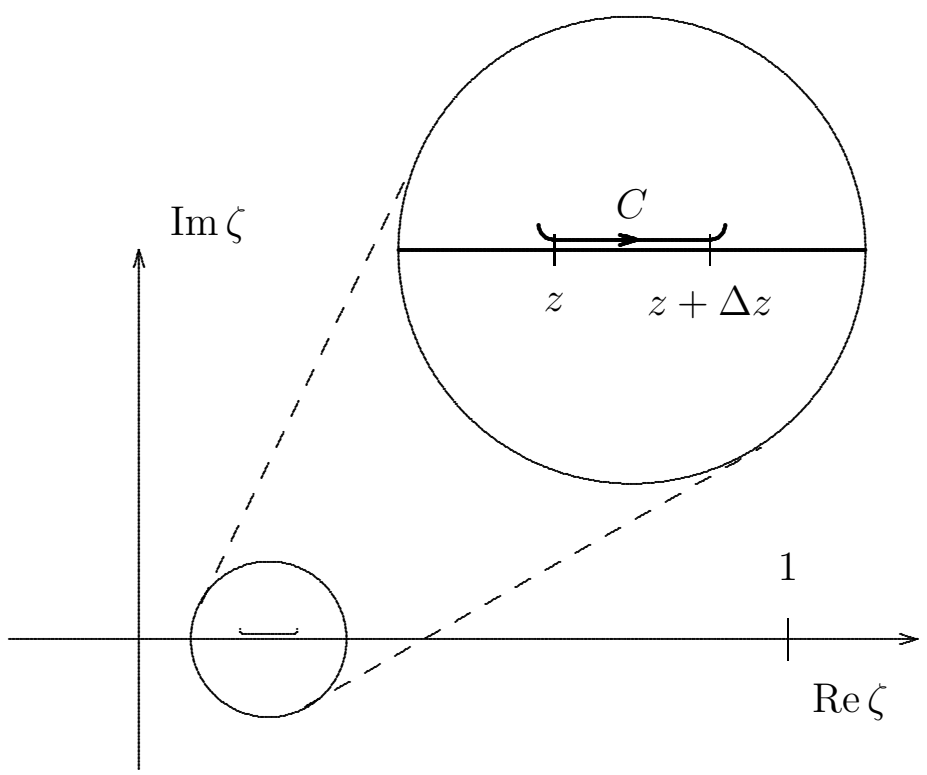

Figure 1. Plot of the integration contour $C$. 
Figure 2

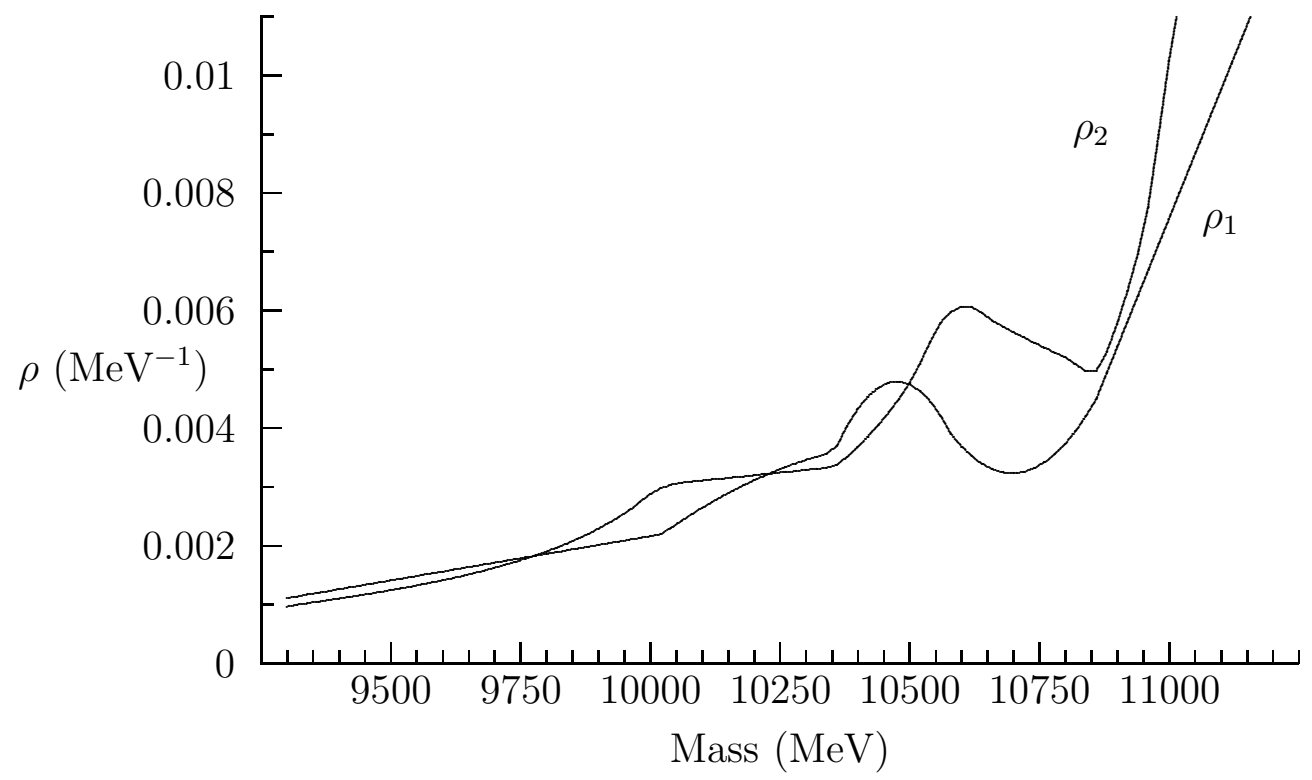

Figure 2. Plot of the densities $\rho_{1}$ and $\rho_{2}$. 
Figure 3

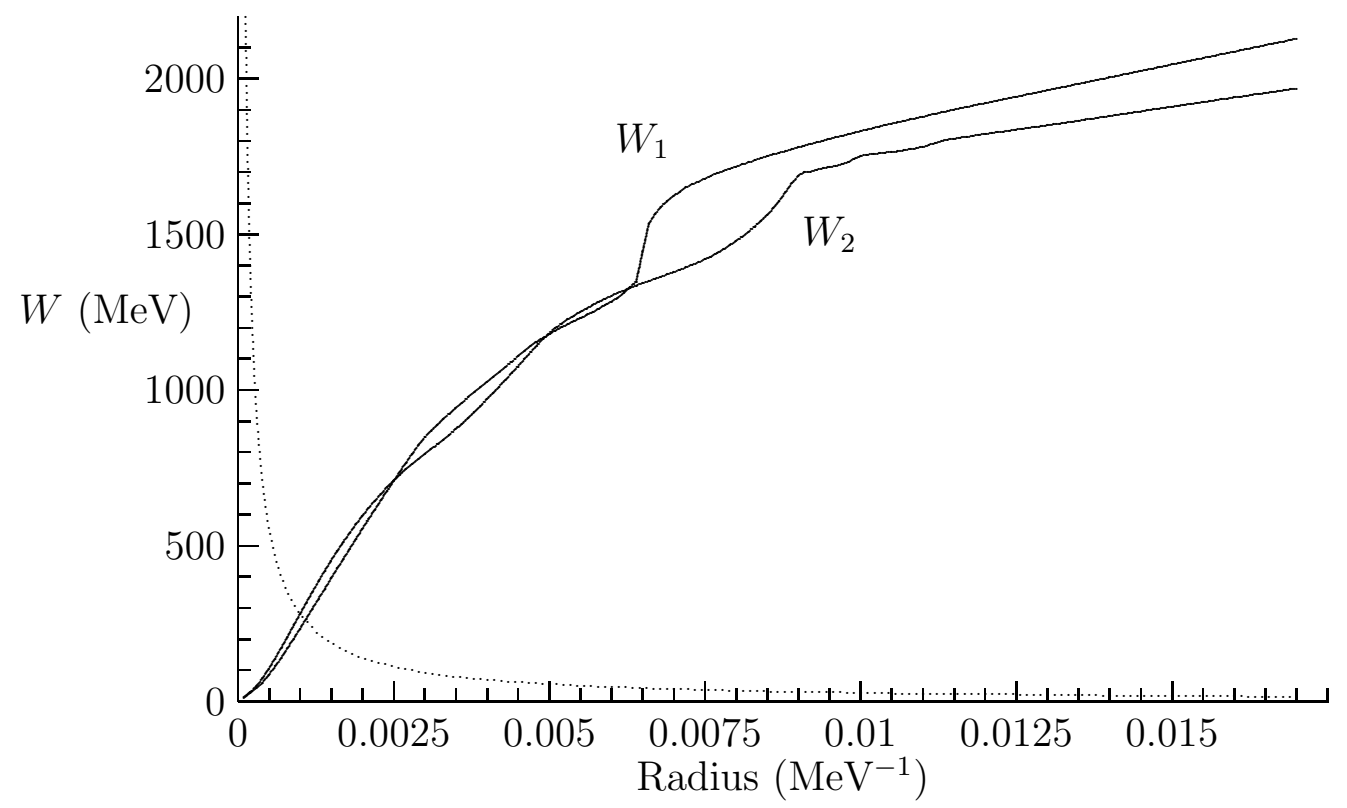

Figure 3. Plot of the confining potentials $W_{1}$ and $W_{2}$. The dotted line is $\alpha_{s} C_{F} / r$. 